\title{
Capillary Flow Properties of Phenolphthalein Poly(ether ether ketone) (PEK-C)
}

\author{
Yingwei DI, ${ }^{\dagger}$ Demin XIE, Rongshun WANG, Maria Rossella NoBILE, ${ }^{*}$ \\ and Domenico ACIERNo* \\ Department of Chemistry, Northeast Normal University, \\ Changchun 130024, People's Republic of China \\ * Department of Chemical and Food Engineering \\ University of Saierno, Italy
}

(Received January 17, 1996)

\begin{abstract}
The capillary flow properties of novel engineering thermoplastic phenolphthalein poly(ether ether ketone) (PEK-C) have been investigated using capillary rheometer. The dependence of viscosity on the wall shear rate and temperature were obtained. The entrance effect was calculated and from which the extensional behavior was also estimated. From the extudate swell ratio the principal normal stress were also evaluated. The melt fracture phenomena were checked and discussed. KEY WORDS Phenolphthalein Poly(ether ether ketone) (PEK-C) / Capillary Flow / Entrance Pressure Drop / Extrudate Swell / Melt Fracture /
\end{abstract}

Phenolphthalein poly(ether ether ketone) (PEK-C), as a new emerging member of the family of engineering thermoplastics, had been becoming more and more applicable in many fields including advanced composite application since it was developed in eighties ${ }^{1}$ because of its high-temperature resistance $\left(T_{\mathrm{g}} \approx 220^{\circ} \mathrm{C}\right)$, excellent mechanical properties, better solubility in certain solvents and more economical cost. Owing to the cardo group attached on its main chain it is an amorphous polymer with exceptional thermal stability. For the industrial interest, the flow behavior of this novel material needs to be well understood in order to determine the proper processing conditions.

It has been amply known that pressure driven flow through tubes and other types of channels is of central importance in experimental rheology and in polymer processing. Not only is this flow used as the basis for the most popular type of melt rheometer, but it is also a flow that occurs often in melt processing, for example in an extrusion die or in the runner feeding an injection mold. In this study we present the results on the investigation of the capillary flow properties on PEK-C melt including flow curves, temperature effect, entrance effect, extrudate swell, and melt fracture.

\section{EXPERIMENTAL}

\section{Materials}

Phenolphthalein Poly(ether ether ketone) (PEK-C) is now commercially available in China. The sample used here are supplied by Xuzhou Engineering Plastics Co. in the form of powder with the reduced viscosity of 0.49 in chloroform at $25^{\circ} \mathrm{C}$ as given by manufacturer and its chemical structure is following:

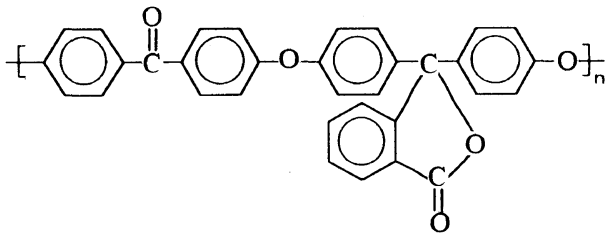

† To whom correspondence should be addressed.
Its molecular weight and molecular weight distribution was measured as $7.25 \times 10^{5} \mathrm{~g} \mathrm{~mol}^{-1}$ and 2.18 with Waters chromatography: $150 \mathrm{CV}$ Gel Permeation Chromatography.

\section{Sample Preparation}

The powder materials were extruded and pelletized twice using a twin-screw extruder with an $L / D$ of 22 and a compression ratio of 23 under conditions of cylinder and die temperature of $320,320,330$, and $350^{\circ} \mathrm{C}$ with screws revolution speed of $40 \mathrm{rpm}$. Before extrusion the material were dried in vacuum oven at $140^{\circ} \mathrm{C}$ over night to erase any moisture and solvent residuals. The reduced viscosity of extrudates was measured again and the molecular weight changes were found to be negligible.

\section{Capillary Characterization}

Melt rheology, die swell and melt fracture were investigated using the CEAST capillary rheometer, Rheoscope 1000 in which the flow of sample is generated by a piston moving down at constant speed in a reservoir above the capillary. The reservoir has a dimension of $9.5 \mathrm{~mm}$ diameter and $166 \mathrm{~mm}$ length. The samples in the form of pellet were dried in the vacuum oven over night before being loaded into the rheometer. All flat dies used for rheology study had a diameter of $1 \mathrm{~mm}$ and the length-to-diameter ratios $(L / D)$ vaired from 5 to 20 . The experimental temperature for extrusion is controlled by the heating sheath around the reservoir and the temperatures of $310,320,330$, and $340^{\circ} \mathrm{C}$ were used in this study at different piston speeds. The relation between pressure, $P$, and volumetric flow rate, $Q$, was measured with the different dies to determine the flow curves of PEK-C. Shear rates from 60.2 to $240.7 \mathrm{~s}^{-1}$ could be obtained. Higher shear rates were precluded because of the load limitation of the rheometer.

Ideal extrudate swell measurements should be performed under the following conditions. ${ }^{2}$ (1) steady isothermal flow; (2) absence of gravitation force as well as interfacial tension; (3) should be in a state of complete elastic recovery. There are several methods of measurement of extrudate swell. ${ }^{2-4}$ In this study the simplest 
method was adopted, i.e., quenching the extrudates in air after they were extruded from capillary and then measuring their diameters at a constant distance of $2 \mathrm{~cm}$ from the exist of capillary at room temperature using a micrometer when they were air frozen. The diameter of the extrudate, $D$, was determined as an average of two measurements at right angles to each other (or as nearly as possible). This was done to compensate for the noncircular cross sections and each time at least 5 samples were used and averaged to get the value of $D$. The ratio $D / D_{0}$, where $D_{0}$ is the capillary diameter was called die swell ratio and used as a measure of die swell.

Onset of melt fracture was simply determined by the observation of the extrudates appearance with naked eye and the occurrence of melt fracture was checked.

\section{RESULTS AND DISCUSSION}

\section{Flow Curves}

The apparent shear stress $\tau_{\mathrm{a}}$ and the apparent shear rate $\dot{\gamma}_{\mathrm{a}}$ at the wall in capillary flow are given by following two equations 5 :

$$
\begin{gathered}
\tau_{\mathrm{a}}=\frac{P \cdot R}{2 L} \\
\dot{\gamma}_{\mathrm{a}}=\frac{4 Q}{\pi R^{3}}
\end{gathered}
$$

where $P$ and $Q$ are pressure drop through capillary and the volumetric flow rate respectively. Figure 1 shows, as an example, the apparent flow curves $\left(\dot{\gamma}_{\mathrm{a}}-\tau_{\mathrm{a}}\right)$ obtained directly from eq 1 and 2 for the data at $320^{\circ} \mathrm{C}$ with different dies.

Due to the entrance pressure loss, the apparent flow curve obtained with a die of smaller $L / R$ locates at higher shear stress side. To obtain real stress at the wall of capillary, $\tau_{w}$, the Bagley end correction is often adopted, ${ }^{6}$

$$
\tau_{\mathrm{w}}=\frac{P}{2\left(\frac{L}{R}+e\right)}=\frac{P-P_{\mathrm{ent}}}{2\left(\frac{L}{R}\right)}
$$

where $e$ is end correction and $P_{\text {ent }}$ is the entrance pressure drop. Both of them can be calculated as the respective intercept of $L / D$ and $P$ axes by extrapolating the straight lines of $P$ versus $L / D$ corresponding to various values of $\dot{\gamma}_{\mathrm{a}}$ to the $P=0$ axis. An example of the Bagley plot is

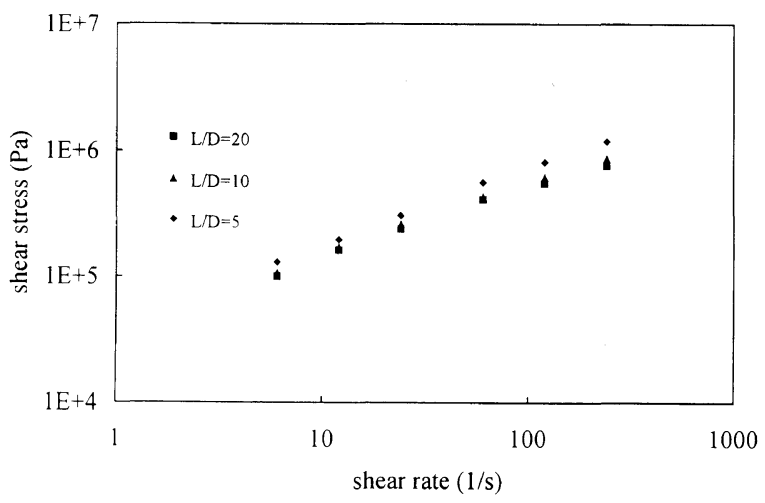

Figure 1. The flow curves for PEK-C at $320^{\circ} \mathrm{C}$ with three dies. shown in Figure 2 for PEK-C at $320^{\circ} \mathrm{C}$. Fine Bagley plots were obtained for all samples. The flow curve $\left(\dot{\gamma}_{\mathrm{a}}-\tau_{\mathrm{w}}\right)$ at $320^{\circ} \mathrm{C}$ by correcting the apparent shear stress $\tau_{\mathrm{a}}$ to the effective wall shear stress $\tau_{\mathrm{w}}$ using eq 3 can be obtained and each flow curve with different $L / D$ unites into one curve independent of $L / D$ (for the reason of clarity they are not shown in Figure 1).

On the other hand, the non-Newtonianity of flow is also needed to be corrected and "the Rabinowitch correction" $"$ is usually used by feeding back the entrance correction into the data to get the plots of $\log$ (wall shear stress, $\left.\tau_{\mathrm{w}}\right)$ versus $\log \left(\right.$ apparent shear rate, $\left.\dot{\gamma}_{\mathrm{a}}\right)$. The slopes of these later plots, $n=\mathrm{d} \log \tau_{\mathrm{w}} / \mathrm{d} \log \dot{\gamma}_{\mathrm{a}}$ which also gave the polymer flow behavior index at various temperatures, were calculated by the regression analysis and the wall shear rates, $\dot{\gamma}_{\mathrm{w}}$ were given as following.

$$
\dot{\gamma}_{\mathrm{w}}=\left(\frac{3 n+1}{4 n}\right) \cdot \dot{\gamma}_{\mathrm{a}}
$$

In the plot of the logarithm of the $\dot{\gamma}_{\mathrm{a}}-\tau_{\mathrm{w}}$, the data do not fall on a straight line, which implies that the melt behavior of PEK-C is neither Newtonian nor power-law. Consequently the values of $n$ can only be calculated by the non-linear regression analysis on the experimental $\log \dot{\gamma}_{\mathrm{a}} v s . \log \tau_{\mathrm{w}}$ plot and shown in Table I.

The true flow curve $\left(\dot{\gamma}_{\mathrm{w}}-\tau_{\mathrm{w}}\right)$ could be obtained by correcting the apparent shear rate $\dot{\gamma}_{a}$ to the wall shear rate $\dot{\gamma}_{w}$ using eq (4) and in Figure 3 they are shown by plotting $\eta$ versus $\dot{\gamma}_{\mathrm{w}}$ at various temperatures in which much clearer flow behavior is shown.

In Figure 3 it is showed that increased shearing

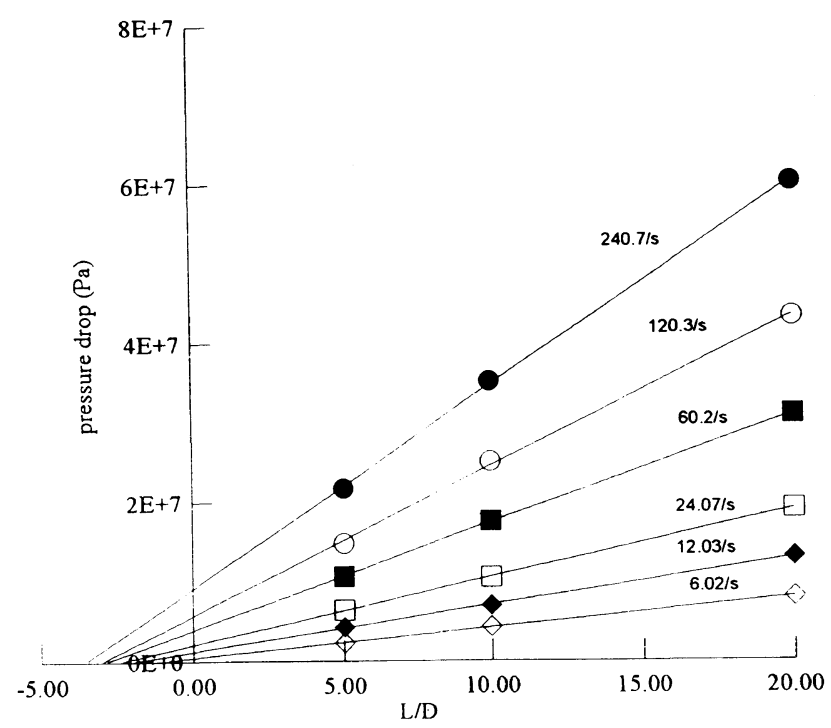

Figure 2. The Bagley entrance correction plot for PEK-C at $320^{\circ} \mathrm{C}$

Table I. The melt flow indexes for PEK-C

\begin{tabular}{ccccc}
\hline $\begin{array}{c}\text { Apparent shear } \\
\text { rate } / \mathrm{s}^{-1}\end{array}$ & $340^{\circ} \mathrm{C}$ & $330^{\circ} \mathrm{C}$ & $320^{\circ} \mathrm{C}$ & $310^{\circ} \mathrm{C}$ \\
\hline 6.02 & 0.9625 & 0.8286 & 0.8 & 0.7471 \\
12.03 & 0.9223 & 0.7949 & 0.7649 & 0.7179 \\
24.04 & 0.8821 & 0.7612 & 0.7298 & 0.6886 \\
60.2 & 0.8289 & 0.7166 & 0.6834 & 0.6499 \\
120.3 & 0.7888 & 0.6828 & 0.6483 & 0.6206 \\
240.4 & 0.7485 & 0.6491 & 0.6132 & 0.5913 \\
602 & 0.6954 & - & - & - \\
\hline
\end{tabular}




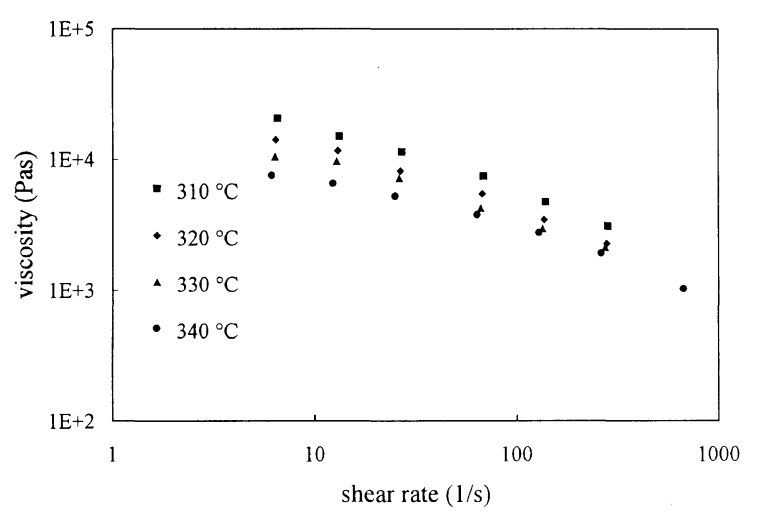

Figure 3. The capillary flow curves for PEK-C at four temperatures.

decreases the melt viscosity as expected like other high performance thermoplastics polymeric materials, e.g., Ultem, PEI,${ }^{9}$ and Victrex 380 , PEEK,${ }^{10}$ which all show the shear-thinning behavior. By rough comparison with the data of Ref. 10 on PEEK, we can observe the viscosity values of PEEK at $350^{\circ} \mathrm{C}$ are in the same order of magnitude as that of PEK-C at $330^{\circ} \mathrm{C}$ in the same shear rate range. So the material used in this study has a better processability than PEEK of Victrex 380 .

Because no significant molecular weight changes were detected after extrusion, so the changes in the viscous behavior of the melt must therefore be due to changes in melt morphology. It has been plausibly postulated $^{11,12}$ that shear modification reduces the number of entanglements in the melt and therefore results in the shear-thinning behavior. The present results could also attributed to the disentanglement of the polymer chains.

From Table I in which the flow behavior indexes $(n)$ are listed, we can observe that at each apparent shear rate the values of $n$ increase with temperature. This also indicates that the pseudoplastic behavior for PEK-C melt are more evident as temperature decreasing the shear rate increasing.

\section{Temperature Dependence}

The temperature dependence of the viscosity of polymer melts can be expressed by the flow activation energy as following.

$$
\eta=A \cdot \exp \left(E_{\mathrm{a}} / R T\right)
$$

The higher the flow activation energy, the more temperature sensitive is the melt. Figure 4 is an Arrhenius type plot showing viscosity-temperature dependence of PEK-C melt at two constant shear stresses and two constant shear rates, respectively. The flow activation energy calculated from these plots are given in Table II. Because the flow behavior of polymer melt is dependent upon shear rate and temperature, the flow activation energy of a polymer melt evaluated in the non-Newtonian region at constant shear stresses is more important in understanding the temperature dependence than that evaluated at constant shear rates. ${ }^{13}$ As shown in Table II the flow activation energy calculated at two constant shear stresses are constant as expected. However, the flow activation energy decreases with increasing the shear rates. For some other polymers, e.g., PVC, when the flow activation energy has been evaluated at a constant shear rate the existence of two types of flow

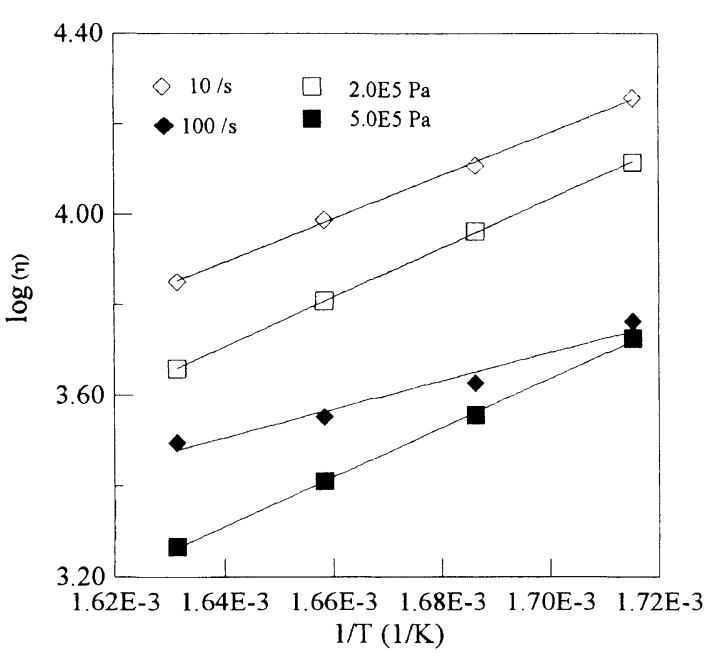

Figure 4. The Arrhenius plot for PEK-C at two constant shear stresses and two shear rates.

Table II. The flow activation energy for PEK-C at two constant stresses and two constant shear rates

\begin{tabular}{ccccc}
\hline & $\tau_{1}=5 \mathrm{E} 5 \mathrm{~Pa}$ & $\tau_{2}=2.0 \mathrm{E} 5 \mathrm{~Pa}$ & $\dot{\gamma}_{1}=10 \mathrm{~s}^{-1}$ & $\dot{\gamma}_{2}=100 \mathrm{~s}^{-1}$ \\
\hline $\begin{array}{c}\text { Activation } \\
\text { energy } / \mathrm{kJ} \mathrm{mol}^{-1}\end{array}$ & 104.5 & 104.4 & 91.7 & 60.2 \\
\hline
\end{tabular}

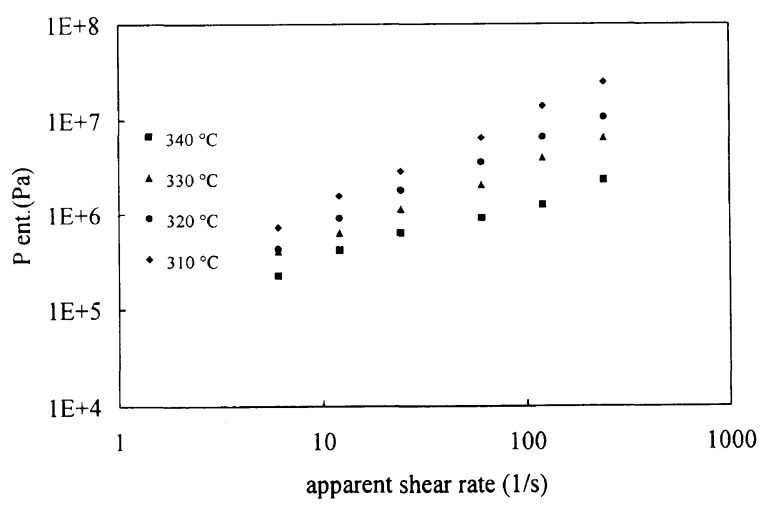

Figure 5. Entrance pressure drop for PEK-C as a function of shear rate at different temperatures.

has been reported by Collins et al. ${ }^{14}$ This is called transitional flow behavior. At constant shear stress, they have seen only a slight evidence to such a flow behavior. However, from our results we could not observe the existence of a two-type flow behavior from either of the Arrhenius plots made at constant stress and shear rate. Hence, it may be understood that the flow transition behavior may not exist in PEK-C used here or it probably takes place outside the temperature and shear rate range used in this study.

\section{Entrance Effect}

The entrance pressure drop values, $P_{\text {ent }}$, from Bagley plots, i.e., plots of $P$ vs. $L / D$ at fixed shear rate, are determined by simple linear least-square fitting. The results are shown in Figure 5 where the variation of the entrance pressure drop with apparent shear rate $\dot{\gamma}_{a}$ at different tempeatures can be observed. $P_{\text {ent }}$ increases with increasing $\dot{\gamma}_{\mathrm{a}}$ and at higher temperature the $P_{\text {ent }}$ 
has a lower value. Large pressure drop at the capillary entrance for polymer melts, compared to that for Newtonian fluids, has been almost exclusively attributed to the elasticity of polymer melts. ${ }^{15}$ It can be said here that the melt elasticity of PEK-C is decreasing with the increase of temperature. For the smallest $L / D$ die $(L / D=5)$ the entrance effects account for $31.7 \%$ of the total pressure drop while for the largest $(L / D=20)$ it is only $10.9 \%$ at temperature of $320^{\circ} \mathrm{C}$ and apparent shear rate of $60.2 \mathrm{~s}^{-1}$. So $P_{\text {ent }}$ is a important parameter characterizing the materials elastic properties.

Since the high degree of tensile extension (stretching along streamline) that occurs at the entrance which also causes the entrance pressure loss, so the entrance pressure drops are also required when using the method of Cogswell ${ }^{16}$ to assess the extensional behavior of the melt. These equations are derived from a simple geometric analysis. He assumes that this pressure drop has two components, one due to shear and one to extension. He further assumes that the extensional strain is sufficiently large that the stored elastic energy resulting from stretching is approximately equal everywhere to some maximum value. The estimated extensional strain rate is given by:

$$
\dot{\varepsilon}_{\mathrm{E}}=\frac{4 \dot{\gamma}_{\mathrm{a}}^{2} \cdot \eta_{\mathrm{a}}}{3(n+1) P_{\mathrm{ent}}}
$$

where $\dot{\gamma}_{\mathrm{a}}$ is the apparent shear rate given in eq 2 and $n$ is flow behavior index as shown in Table I. The extensional stress is given by:

$$
\sigma_{\mathrm{E}}=\frac{3}{8}(n+1) \Delta P_{\mathrm{ent}}
$$

Thus we see that

$$
\eta_{\mathrm{E}}=\frac{9(n+1)^{2}\left(P_{\mathrm{ent}}\right)^{2}}{32 \eta_{\mathrm{a}} \dot{\gamma}_{\mathrm{a}}^{2}}=\frac{\eta_{\mathrm{a}}}{2}\left[\frac{\dot{\gamma}_{\mathrm{a}}}{\dot{\varepsilon}}\right]^{2}
$$

The results for the PEK-C used in this study are shown in Figure 6. It can observed that the extentional stress is an increasing function of the extensional strain rate like the steady shear behavior showed before. This also agrees with the behavior described by the others ${ }^{17}$ for application of Cogswell's method to the other polymers. There has also been a report ${ }^{18}$ on the agreement between the values of $\eta_{\mathrm{E}}$ calculated from this equation for several polymers and the true extensional viscosity determined using elaborate extensional rheometers,

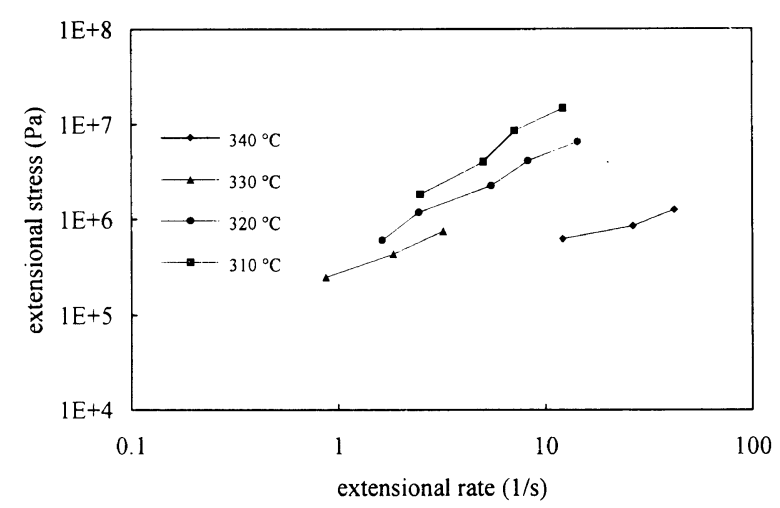

Figure 6. The extensional stress versus extensional rate for PEK-C at different temperatures. however, this agreement only covered narrow ranges of strain rate and for some other exceptional resins and strain rate there was no quantitative correlation between the two functions. So we could only conclude intuitively here that Figure 6 at least showed the general and qualitative trend of extensional behavior for PEK-C.

\section{Extrudate Swell}

The extrudate swell is defined as the value of extruded sample diameter divided by the value of die diameter. Figure 7 shows the variation of die swell ratio $D / D_{0}$ with apparent shear rate $\dot{\gamma}_{\mathrm{a}}$ using temperature as a parameter. It can be observed that the die swell ratios of PEK-C increase monotonously with increasing shear rate. As increasing the length to diameter ratio, the melt swell is reduced as shown in Figure 8. In general, these trends are followed by most thermoplastic resins. ${ }^{19}$ But there also some exception, e.g., poly(vinyl chloride) whose variation of die swell ratio with shear rate sometimes shows a maximum or a minimum. ${ }^{16}$ Figure 7 also exhibit decreasing of die swell ratio with the increase of temperature. This can be attributed to the decrease of elasticity as the increase of temperature. For Newtonian fluid the ratio is about 1.13 , but for PEK-C they are larger than that. These large swell ratios are a manifestation of the molecular orientation that is generated by the flow in the die.

The principal normal stress difference $\left(\tau_{11}-\tau_{22}\right)$ were caluclated from the die swell ratio and wall shear stress according to Tanner's equation ${ }^{20}$ :

$$
\tau_{11}-\tau_{22}=2 \tau_{\mathrm{w}}\left[2\left(D / D_{0}\right)^{6}-2\right]^{1 / 2}
$$

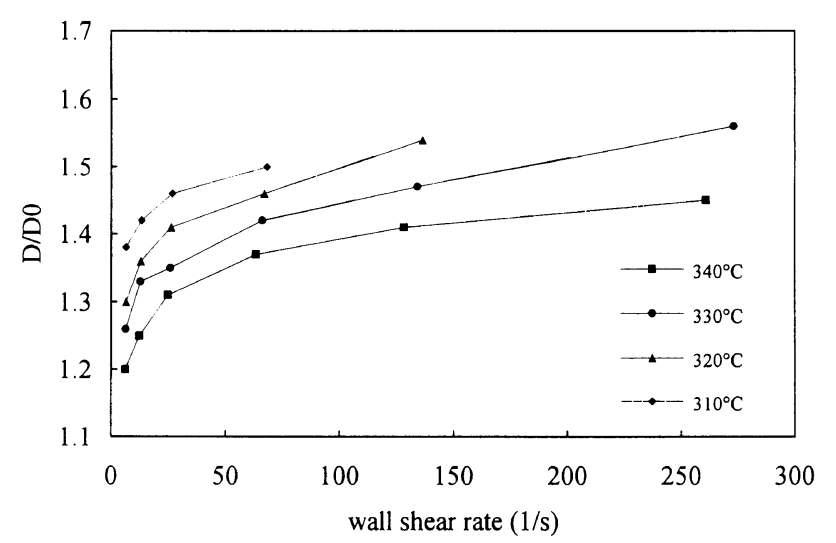

Figure 7. The melts swell plot for PEK-C at four temperatures with a die of $L / D=20$.

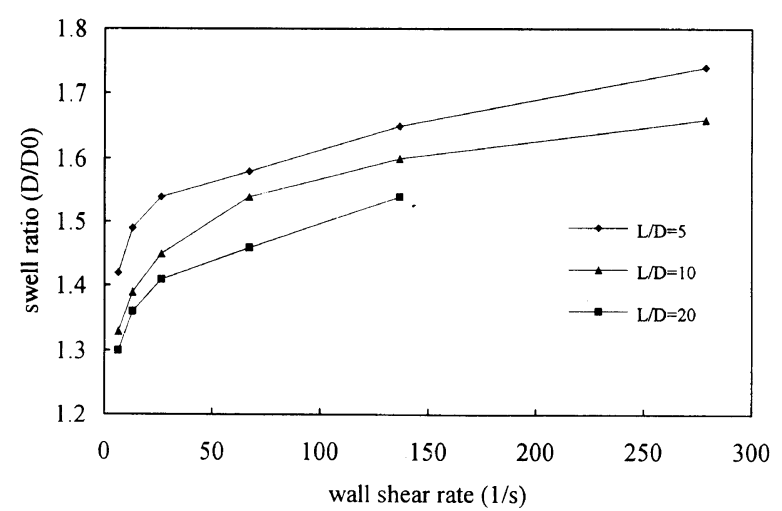

Figure 8. The melt swell ratio plot for PEK-C at $320^{\circ} \mathrm{C}$ with different capillary geometries. 


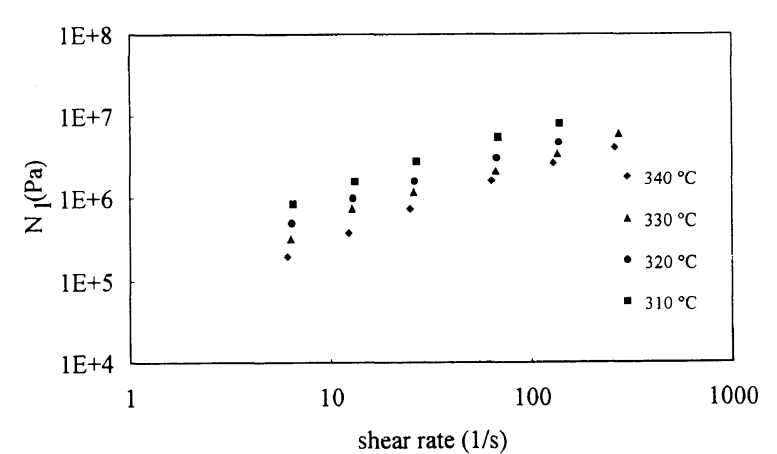

Figure 9. Principal normal stress differences as a function of wall shear rate for PEK-C.

The calculated principal normal stress difference as a function of shear rate are shown in Figure 9 revealing that the $\tau_{11}-\tau_{22}$ of PEK-C increases with shear rate and decreases with temperature. Actually the principal normal stress difference could be measured with the cone/plate rotational rheometer which is limited in low shear rate range and measurement in high shear rate range becomes experimentally difficult. So the results got here at high shear rate range according to the empirical equation are useful for actual processing design.

\section{Melt Fracture}

Melt fractures occurred at each experiment with different die and temperature were checked with the naked eye. The experimental observation showed that the melt fractures in this study had the appearance of a helical screw thread. It has been suggested ${ }^{21}$ that this type of distortion results from the abrupt change in the boundary condition at the exit of the die, which causes a high degree of stretching in the surface layer of the melt as it leaves the die. This stretching produces tensile stresses that can exceed the strength of the melt, leading to surface fracture. But later careful studies ${ }^{22}$ of extrudates of linear polyethylene suggest that the onset of this surface fracture is accompanied by the occurrence of wall slip in the capillary. Thus, while this type of distortion is clearly an exit effect, it may be associated with slip flow in the capillary.

Melt fractures often occur at a critical shear stress or a critical shear rate depending on the different dies. In this study, a critical apparent pressure drop through the capillary was found to be about $6.0 \times 10^{7} \mathrm{~Pa}$ on which the onset of noticeable melt fracture occurs. No critical shear rate was found probably because this shear rate is out of the shear rate range used in this study.

\section{CONCLUSION}

The capillary flow properties of the novel engineering thermoplastic PEK-C have been investigated. It showed a similar pseudoplastic behavior as the other engineering thermoplastics, e.g., PEEK and PEI (Ultem) and the steady shear viscosity values are lower than those of PEEK in the same shear rate and temperature ranges. The dependence of viscosity on the temperatures does not change significantly with shear stress, but decreases with shear rate and the flow transitional behavior have not been observed in this study. The entrance effect from the different dies are obtained and found to increase as the temperature decreasing. According to the entrance pressure drop results, the extensional behavior was estimated. The die swell ratios $D / D_{0}$ of the melts increase with shear rate and decrease with the length to diameter ratio. The principal normal stress at high shear rate were evaluated using Tanner's equation. The critical apparent pressure of the melt at which melt fractures begin to occur was obtained. The critical shear rate was not observed.

Acknowledgment. One of the authors, Yingwei Di, is grateful to Prof. Luigi Nicolais, University of Napoli, Italy, for his financial support on carrying out part of this work.

\section{REFERENCES}

1. H. Zhang, T. Chen, and Y. Yuan, Chinese patent, 85108751.5 (1985).

2. L. A. Utraki, Z. Bakerdjian, and M. R. Kamal, J. Appl. Polym. Sci., 19, 48 (1975).

3. W. M. Graessley, S. D. Glasscock, and R. L. Grawley, Trans. Soc. Rheol., 14, 4 (1970).

4. C. D. Han and T. C. Yu, AIChE J., 17, 1512 (1971).

5. J. M. Dealy and K. F. Wissbrun, "Melt Rheology and Its Role in Plastics Processing," Van Nostrand Reinhold (VNR), New York, N.Y., pp 298-300.

6. J. M. Dealy and K. F. Wissbrun, "Melt Rheology and Its Role in Plastics Processing," Van Nostrand Reinhold (VNR), New York, N.Y., p 319.

7. J. M. Dealy and K. F. Wissbrun, "Melt Rheology and Its Role in Plastics Processing," Van Nostrand Reinhold (VNR), New York, N.Y., p 303.

8. J. M. Dealy and K. F. Wissbrun, "Melt Rheology and Its Role in Plastics Processing," Van Nostrand Reinhold (VNR), New York, N.Y., p 163.

9. L. Incarnato, M. R. Nobile, and D. Acierno, Makromol. Chem., Macromol. Symp., 68, 277 (1993).

10. A. Mehta and A. I. Isayev, Polym. Eng. Sci., 31, 971 (1991).

11. A. Rudin and H. P Schreiber, Polym. Eng. Sci., 23, 422 (1983).

12. P. J. R. Lebans and C. Bastiaansen, Macromolecules, 22, 3322 (1989).

13. R. S. Porter, J. Whan, and F. Johnson J. Polym. Sci., C, 15, 365 (1966).

14. E. A. Collins and C. A. Krier, Trans. Soc. Rheol., 11, 225 (1967).

15. M. Fujiyama and Y. Kawasaki, J. Appl. Polym. Sci., 42, 467 (1991).

16. F. N. Cogswell, Polym. Eng. Sci., 12, 64 (1972).

17. M. V. Prooyen, T. Bremner, and A. Rubin, Polym. Eng. Sci., 34, 570 (1994).

18. H. M. Laun and H. Schuch, J. Rheol., 33, 119 (1989).

19. C. D. Han, M. Charles, and W. Philippoff, Trans. Soc. Rheol., 14, 19 (1970).

20. R. I. Tanner, J. Polym. Sci. A-2, 14, 2067 (1970).

21. N. F. Cogswell, "Polymer Melt Rheology," John Wiley \& Sons, New York, N.Y., 1981, p 101.

22. D. S. Kalida and M. M. Denn, J. Rheol., 31, 815 (1987). 